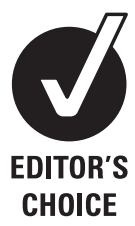

$10.1136 /$ ebm 1094

Editor, Evidence-based Medicine

Correspondence to

Richard Saitz

Section of General Internal Medicine, Boston Medical Center, Boston University Schools of Medicine and Public Health, 801 Massachusetts Avenue, 2nd floor (Crosstown 2), Boston, MA 02118-2335, USA; rsaitz@bmjgroup.com

\title{
Evidence-based medicine: time for transition and translation (to practice)
}

\section{Richard Saitz}

It is a time of change for evidence-based medicine (EBM, the field) and for $E B M$ (the journal). With this issue of EBM I become its new Editor; I have assembled an editorial board consisting of primary care and internal medicine practitioners with expertise in both critical appraisal and clinical practice (general practice, family medicine, internal medicine, paediatrics and obstetrics and gynaecology). The main purpose of the journal remains the same: to briefly summarise and critically appraise articles that appear in the peer-reviewed health literature and are likely to be valid and relevant for clinical practice. The world of healthcare, however, has changed since EBM arrived on the scene.

The past 20 years have seen the birth of $E B M^{1}$ and its adolescence, during which grown-ups in medicine either seemed skeptical about the upstart movement or described it as nothing new. I witnessed this development and took on a role that included its teaching and practice. Journal club during my residency (postgraduate training) usually involved an article that was chosen for little apparent reason (not because of a question it was likely to address). The article was then shredded (at least figuratively) by a resident, and then specialist commentary based on clinical experience and expertise was sprinkled in. When the concept of EBM appeared in the literature, and I had attended a teaching-EBM workshop at McMaster University, my eyes were opened to how much more useful the medical literature could be. The need to handle the information explosion in an organised fashion was great. EBM served as a framework for selecting and evaluating articles. It became a useful tool for keeping up with the literature and a solid basis for practicing medicine.

That science is now mature, EBM firmly in middle age, with experts in searching and critical appraisal all around. And research methodology has advanced, perhaps even outstripping its clinical utility at times. But many challenges remain for EBM on using it to its fullest potential. ${ }^{2}$ It is still difficult to keep up with the literature, and for many, critical appraisal is an elusive skill. EBM (the journal) helps with these issues. But the real challenges are how to translate evidence into policy and practice. Such translation involves values and preferences. Recent controversies about breast cancer screening and vaccines do not appear to have been as much about evidence as they were about values, preferences, beliefs and translation of evidence into practice and policy (including issues of cost and payment). ${ }^{34}$ Decisions about what care to pay for vary based on data beyond efficacy, as I recently was reminded during a visit to meet with British Medical Journal Group editors in the UK where the news was about how varicella vaccine was not paid for by the National Health Service; in contrast, the vaccine is widely disseminated, considered to be the standard of care and covered by health insurance in the USA. Clinicians and patients (and policymakers) want good evidence, but they also want to know what to do with it. Critical appraisal of an article seems simple in comparison, and practice guidelines help only a little bit in the clinic with individuals.

A few more examples are in order. In this issue of EBM, the reader will find a commentary on the results of a randomised trial of dutasteride for preventing prostate cancer. ${ }^{5}$ The study found efficacy for men 50-75 years of age who had had a recent negative prostate biopsy and prostate specific antigen (PSA) level of 2.5 (3.0 if age $\geq 60$ ) to $10.0 \mathrm{ng} / \mathrm{ml}$. The commentator concludes that the effects of the medication are clinically relevant and that drugs in this class should be considered for men at high risk (eg, like those in the trial). I come to different conclusions from the same evidence. I would emphasise that the effect of dutasteride was limited to low grade cancers, and that we don't know whether prevention of such cancers (often the focus of over-identification and overtreatment) will improve morbidity or reduce mortality. In that context the 5\% absolute risk reduction seems of unclear clinical relevance (follow-up biopsies were part of the protocol, not based on symptoms or PSA level). An editorialist wrote that the drug did not prevent prostate cancers; rather it temporarily shrank tumours with low potential for being lethal. ${ }^{6}$ He also pointed out that the suppressed PSA levels might delay diagnosis and treatment until the development of high-grade disease. The interpretation of such results depends on much beyond the evidence per se.

I hope international readers will forgive me for a parochial example. I use it because it illustrates the challenges for EBM well. Jacoby Ellsbury is a popular baseball player with the Boston Red Sox. In April this year, another player slammed into him knee first, and a plain radiograph was negative. ${ }^{7}$ He was put on the 'disabled list' because of pain in his chest to return when better. Eleven days after the injury he had a CT scan, which he said was done at his request. The CT scan found non-displaced hairline rib fractures, leading a sportswriter to write that "it wasn't just a contusion, as the team discovered yesterday." The team physician pointed out that the test hadn't changed anything - the treatment was the same (rest) - and the player could return when the symptoms subsided. We know a CT scan can identify fractures not seen on plain films. But what is the value of that information? It was of no value to the team physician, but the player (and his fans) felt it was valuable in explaining the duration of symptoms. In fact they seemed surprised that a physician would withhold such testing. Ellsbury said "I'm glad I went about it and did it just to kind of get some closure in what's going on."

What will we do here at EBM to help readers keep up and to address the transition of EBM into middle age? 
Here is my plan, and I hope to hear from you about it, and whether or not it is meeting your needs.

1. From among the numerous potentially valid peerreviewed studies published, we will summarise and comment on those most likely to have clinical relevance for medicine practiced by general practitioners, family physicians and internists.

2. We will combine a summary of the original article along with commentary. Original article abstracts can be found online through the link provided. We will summarise the context, key methodological features and results in a structured commentary with subheadings that allow readers to go directly to the section they seek. Experts will provide their view on implications for practice. Our editorial board will peer-review these commentaries.

3. We will include occasional EBM-relevant editorials and perspectives either about articles summarised or about broader issues.

4. EBM intends to be a home for 'EBM-ers', those who teach, study and practice EBM. To that end we invite systematic reviews, EBM teaching and research methods articles, 'primer' articles focusing on EBM tools and concepts and an occasional review of what has appeared elsewhere in the literature relevant to EBM (an EBM Roundup).

In addition to this content, I also recognise that, while the paper journal is certainly endearing and has its many fans and users (it will continue), electronic publishing makes it even easier to keep up with the literature through $E B M$. We will make commentaries available online when they are ready, and readers will be able to receive alerts and read them in smaller boluses more frequently if that is their preference.

EBM is at a crossroads, a transition from searching, finding, appraising and keeping up, to translating evidence into policy and practice. It is time to enter the second 20 years during which we will no doubt see EBM fulfil even more of its promise. Hopefully EBM will continue to be a useful tool and home for all of you during this next phase. Our plans should help with that as should your comments and recommendations.

\section{References}

1. Guyatt G. Evidence-based medicine. ACP J Club (Ann Intern Med) 1991;14(Suppl 2):A-16.

2. Guyatt G, Cook D, Haynes B. Evidence based medicine has come a long way. BMJ 2004;329:990-1.

3. When evidence collides with anecdote, politics, and emotion: breast cancer screening. Ann Intern Med 2010;152:531-2.

4. Retraction - Ileal-lymphoid-nodular hyperplasia, non-specific colitis, and pervasive developmental disorder in children. Lancet 2010;375:445.

5. Vickers A. Dutasteride reduces incident prostate cancer in men after negative prostate biopsy. Evid based Med. Published Online First: 2010 Jun 8. [Epub ahead of print].

6. Walsh PC. Chemoprevention of prostate cancer. $N$ Engl J Med 2010;362:1237-8.

7. Benjamin A. New tests: Ellsbury Has 4 Fractured Ribs. The Boston Globe. 2010. http://www.boston.com/sports/baseball/ redsox/articles/2010/04/23/new_tests_ellsbury_has_4_ fractured_ribs/ (Accessed 31 May 2010). 